\title{
Targeted nucleases: spreading the joy
}

\author{
Methods for targeted genome editing are developing at a fast pace. Are there barriers to their \\ widespread use in research?
}

With the reports earlier this year of RNA-based gene editing, we are now at the third wave of programmable nucleases for targeted genome modification. These tools are used to cut genomic DNA at a defined site, stimulating repair that can result in mutations or in the precise replacement or addition of sequence.

The new RNA-guided endonucleases (RGENs) are directed to their target sites by a complementary RNA molecule. In contrast to previous tools, zinc-finger nucleases (ZFNs) and transcription activator-like effector nucleases (TALENs), the RGEN nuclease component itself does not require re-engineering to target a new sequence (see Research Highlights, page 189).

For each of these new tools, the time it has taken to advance from early theoretical suggestions to actual proof-of-principle demonstrations of gene editing seems to get progressively shorter. For RGENs, the pace (a matter of months) has been dizzying.

And yet, the published literature on even the maturer tools-ZFNs were shown to cleave site specifically in cells more than a decade ago, TALENs about two years ago-consists mainly of proof-of-principle reports. We know that targeted nucleases can be used to make mutations and to precisely change or introduce sequences into endogenous genes in cultured cells and in many organisms. But, with some exceptions, papers that report the use of targeted nucleases do not (yet) apply them to answer biological questions.

In part, this is a matter of time. Use of the first tools, the ZFNs, has spread relatively slowly because they were initially expensive to buy and proved difficult for many researchers to make themselves. It is likely that these early experiences have made some scientists gun-shy about adopting targeted nucleases in spite of the fact that prices have come down substantially and design methods have improved.

TALENs, which are easier to design and make in individual laboratories, have not been around as long. Although constructs for assembling TALENs are available through Addgene (the tools themselves are also commercially available), the need to obtain whole libraries of plasmids for assembling TALENs may be a deterrent for some. Finally, though TALEN assembly works well by many accounts, making good TALENs and applying them to biology still takes more than the wave of a magic wand.

Aside from purely technical considerations (cloning repetitive sequences, for instance), a project based on targeted gene editing involves more than obtaining tools

that cut at the desired site. One must deliver nucleases to the cell type of interest at high enough levels. The nucleases must cut in that cell type or organism with sufficient efficiency and specificity, and low enough toxicity, that clonal cell lines or transgenic organisms with the desired change can be identified without onerous levels of screening. Barriers such as these, typical of any research project, are hardly ever insurmountable. But they take time and effort to cross and the research project has only then really begun.

Paradoxically, the rapid pace of methods development for targeted genome editing could itself prove intimidating for a potential user: in addition to new nucleases, reports of improved design methods for the individual platforms are still coming fast and furious. The elegance of targeted endogenous gene modification notwithstanding, there are often alternative, if sloppier, ways to achieve a similar outcome. Taken together, the activation barrier to set up this methodology could, for some, prove to be high.

Despite the potential difficulties, researchers are clearly taking active notice of these tools. TALEN kits distributed by Addgene number in the thousands and RGEN kits are already being requested too. The first joint conference of developers and potential users in synthetic biology will be held as part of the Keystone series next month. And core facilities to make TALENs are already running at more than one institution, at least in the United States. Such facilities could help streamline the first step of a gene editing project-the choice, design and assembly of the tool-particularly for laboratories that cannot routinely afford to buy them.

There are systems for which there are no good alternative tools and the rewards for adopting targeted nucleases will be great. These include model organisms such as the zebrafish, for which the possibility to modify and tag endogenous genes opens new doors, as well as nonmodel organisms such as the monarch butterfly, for which reverse genetic tools previously did not exist. For studies of gene regulation, or functional studies in which precise protein levels or genomic context are important, these tools will be invaluable. Even where established methods exist-mouse gene targeting, for instance-the efficiency of nuclease-targeted modification could in some cases make it the more effective choice.

Targeted nucleases are surely snipping away at biologically interesting sequences in many laboratories around the world. It should not take too long before these efforts begin to come to fruition. 